\title{
Spatiotemporally Controllable Plasma Lattice Structures in Dielectric Barrier Discharge
}

\author{
Weili Fan, ${ }^{1,2,3, *}$ Zhengming Sheng, ${ }^{2,3,4,5}$ Wei Dang, ${ }^{1}$ Yueqiang Liang, ${ }^{1}$ Kuangya Gao, ${ }^{1}$ and Lifang \\ Dong ${ }^{1}$ \\ ${ }^{1}$ College of Physics Science and Technology, Hebei University, Baoding 071002, China \\ ${ }^{2}$ Key Laboratory for Laser Plasmas (MoE) and School of Physics and Astronomy, Shanghai Jiao Tong University, \\ Shanghai 200240, China \\ ${ }^{3}$ Collaborative Innovation Center of IFSA (CICIFSA), Shanghai Jiao Tong University, Shanghai 200240, China \\ ${ }^{4}$ SUPA, Department of Physics, University of Strathclyde, Glasgow G4 ONG, UK \\ ${ }^{5}$ Tsung-Dao Lee Institute, Shanghai Jiao Tong University, Shanghai 200240, China
}

(Received 15 January 2019; revised manuscript received 26 March 2019; published 25 June 2019)

\begin{abstract}
A method to generate spatiotemporally controllable plasma lattice structures via dielectric barrier discharge is proposed by utilizing a latticed water electrode. A variety of plasma lattice structures including triangle, hexagon, honeycomb, and complex superlattices are obtained by changing three parameters including the applied voltage, the gas pressure, and the gas compositions. Moreover, these plasma lattices can be quickly reconstructed, allowing for active control both in space and time. Two-dimensional particle-in-cell simulations are carried out to demonstrate the formation of plasma structures under different voltages, which are in good agreement with experimental observations. Our method provides a unique way for the fabrication of controllable plasma lattice structures, which may enable tunable control of microwave radiation for wide applications.
\end{abstract}

DOI: 10.1103/PhysRevApplied.11.064057

\section{INTRODUCTION}

Photonic crystals (PCs) are commonly used for manipulating electromagnetic radiation of different frequency ranges [1,2]. Currently, one of the most attractive issues in this field is how to fabricate tunable photonic crystals, allowing for real-time and on-demand control of the photonic band structures. It is particularly interesting to realize rapid reconstruction of complex structures such as the superlattices, which may show band gaps a few times larger than a simple lattice $[3,4]$. So far, there have been some proposals to make tunable PCs via mechanical, thermal, biological, and optofluidic methods [5-8]. Recently, increased efforts have been made using plasma photonic crystals (PPCs) as a special "meta"-material by use of the plasma dispersive properties and the active temporal switching capabilities [9-27]. These have led to some dynamic devices for electromagnetic waves, such as the plasma lens, plasma antenna, plasma stealth aircraft, and so on.

Generally, four methods are implemented to produce tunable PPCs in experiments. First, an array of metal electrodes or an array of discharge tubes are utilized to generate periodic plasma columns whose symmetries are

\footnotetext{
*fanweili@hbu.edu.cn
}

right commensurate with the artificial structures [10-15]. Second, PPCs are fabricated either by inserting an arrangement of solid rods (dielectric or metal) into the plasma background, or in reverse, producing periodic plasma rods in a solid background $[17,18]$. Third, PPCs are achieved by introducing the plasma elements into a crystal cavity structure as point defects [19-21]. Recently, an alternative type of transient photonic crystals has been suggested by counter propagating two high-power laser beams [22-25], which have the band gaps near the driving laser wavelength. For these methods, the tunable responses of PPCs can be achieved by either changing the plasma density (or gas pressure) or the discharge geometric configurations. However, the geometric configurations of PPCs, such as the symmetry or lattice constants of the structures, are normally immutable once the PPCs have been fabricated. So far, it remains a significant challenge to develop a robust fabrication scheme to achieve a flexible and temporally controllable transformation between different symmetries of PPCs.

By using a dielectric barrier discharge (DBD) system with two water electrodes, a variety of plasma lattice structures were obtained in our previous studies [26,27], which can potentially be used as plasma photonic crystals. These structures are formed due to selforganization of the filaments initiated between two flat electrodes. One can switch 
the structures from one to another by changing the discharge parameters. The high nonlinearity of the system facilitates the rich diversity of the lattices, but it imposes a challenge to the control of the plasma structures. Indeed, it is not trivial to give preference to a certain symmetry or a specific superlattice. It is highly demanding to improve the stability and controllability of the PPCs while making diverse structures.

In this paper, we propose and demonstrate an effective method to generate spatiotemporally controllable plasma lattice structures in DBD with a uniquely designed water electrode with a certain mesh array, which may be applied to generate PPCs. A variety of plasma lattice structures, including diverse superlattices, are obtained. By changing three parameters including the applied voltage, the gas pressure, and gas compositions, various plasma lattices with different symmetries can be dynamically constructed. Furthermore, two-dimensional particle-in-cell (PIC) simulations are carried out to demonstrate the formation of filamentary structures with an array of electrodes, which are in agreement with experimental observations.

\section{EXPERIMENTAL METHOD AND SIMULATION MODEL}

The schematic diagram of our DBD setup is illustrated in Fig. 1. Two cylindrical containers with diameters of $7.5 \mathrm{~cm}$ filled with tap water are used as the electrodes [Fig. 1(a)]. The water also serves as a coolant and transparent media for observation and measurement of discharge filaments. A metallic ring is immersed in each container and connected to a sinusoidal ac power supply. The water electrodes are sealed with glass plates with a thickness of $1.5 \mathrm{~mm}$. A meshed template of different symmetries is introduced into the left electrode, which is pasted on the inner wall of the glass plate to

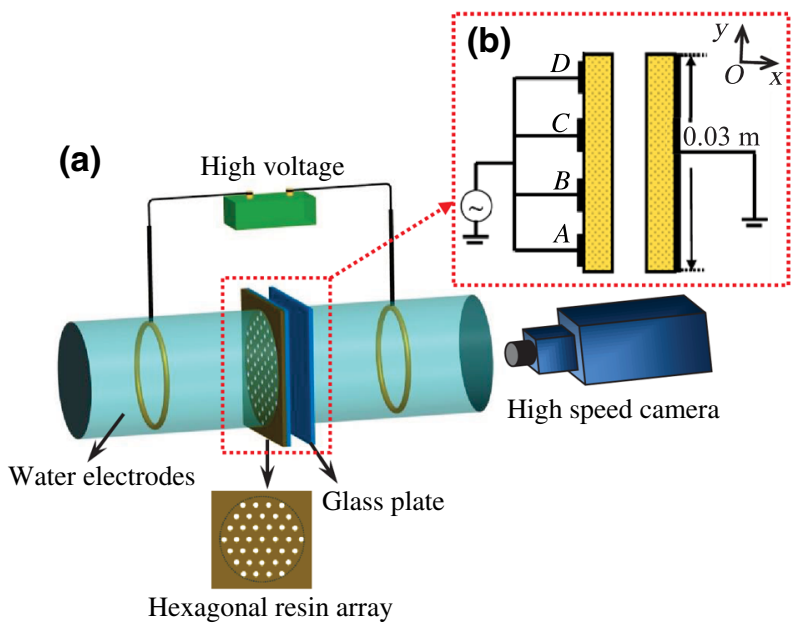

FIG. 1. Schematic diagrams of DBD setup with a latticed water electrode in the experiment (a) and in the numerical model (b). produce a lattice potential. Here, a hexagonal meshed template with a lattice constant of $8.7 \mathrm{~mm}$ is selected, which is fabricated by punching circular holes with diameters of $5 \mathrm{~mm}$ on a 5 -mm-thick acrylic plate. The right electrode is homogeneous and grounded. The whole cell is placed in a big chamber whose gas pressure and gas composition can be changed. The voltage waveform and current waveform are detected using a high-voltage probe (Tektronix P6015A 1000×) and a current probe (Tektronix TCP0030A), respectively, which are recorded using a digital phosphor oscilloscope (Tektronix TDS3054B). An intensified charge-coupled device camera (pco. Dimax 9000000207) is applied to record frames from the end view of the electrodes.

In accordance with the experimental configuration, a numerical model is established [Fig. 1(b)]. The discharge is simulated by using the PIC code $2 \mathrm{~d} 3 \mathrm{v}$ X-Windows Object Oriented Particle-in-Cell code (XOOPIC) complemented with Monte Carlo collisions (PIC MCC) [28-32]. Such simulations are capable of providing a selfconsistent, fully kinetic description of plasma systems, including the nonlocal kinetic effects and the evolution of the electron velocity distribution functions. A two-dimensional $(x-y)$ bounded electrostatic model is employed. The model consists of two parallel electrodes covered with 0.5 -mm-thick dielectric layers. The left electrode is a one-dimensional array with four segments, while the right electrode is a plate that is grounded. The length of each segment and the distance between any two adjacent segments are $4.2 \mathrm{~mm}$. The ion-induced secondary electron emission coefficient (SEEC) on the surfaces of the dielectrics is set as 0.4. A sinusoidal ac voltage is applied to the lattice electrode with a frequency of $f=200 \mathrm{kHz}$. The Dirichlet boundary conditions are adopted for the electrodes. For the top and bottom sides, the Neumann boundary conditions are used where the charged particles, which strike on the boundaries, will be deleted. The simulations track electrons and $\mathrm{He}^{+}$ions while the distribution of background neutrals is assumed to be time independent and uniform in space. Elastic, excitation, and ionization collisions between electrons and neutrals are taken into account via the Monte Carlo method. Moreover, the elastic scattering and charge exchange collisions between ions and neutrals are considered. The discharge is sustained in helium gas at a pressure of 200 Torr, and the gas gap is $0.5 \mathrm{~mm}$. The initial densities of the electrodes and $\mathrm{He}^{+}$ions are set as $10^{15} \mathrm{~m}^{-3}$. This basic model provides a qualitative demonstration on the formation of filamentary structures.

\section{FORMATION OF PLASMA LATTICE STRUCTURES}

Figure 2 presents the distributions of the filaments obtained in both experiment and simulation upon increasing the applied voltage. The experimental observation [row 


\section{(A)}
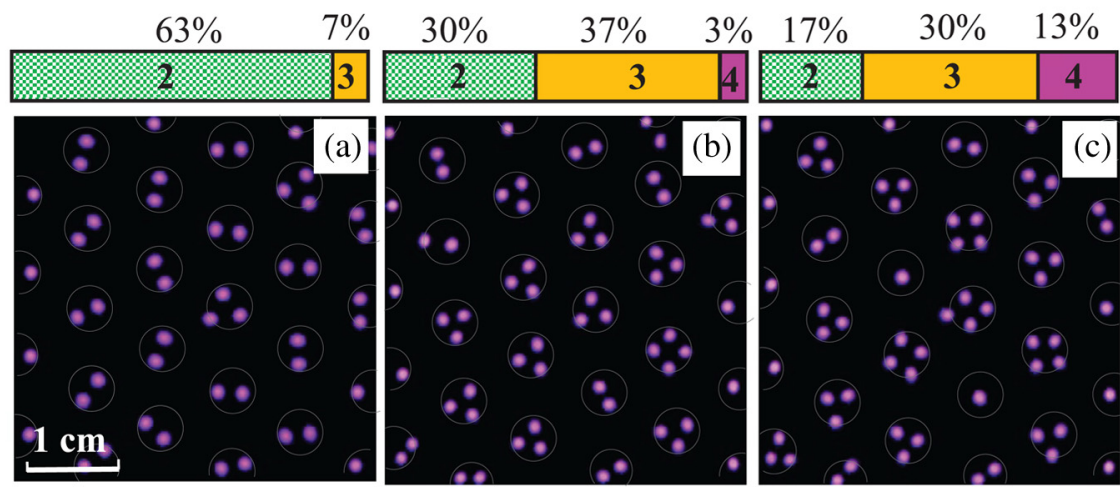

\begin{tabular}{|c|c|c|c|}
$10 \%$ & $3 \%$ & $20 \%$ & $10 \%$ \\
\hline
\end{tabular}

(B)
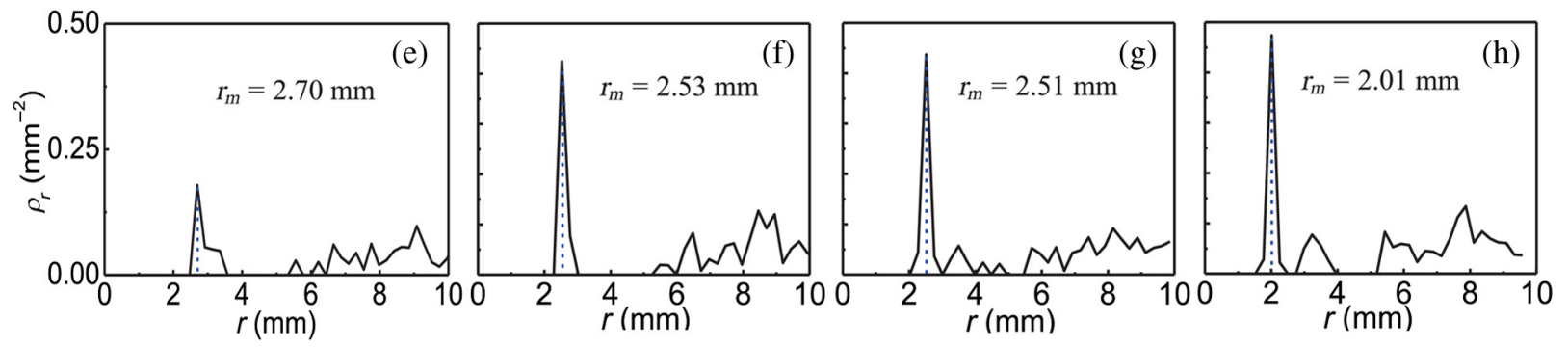

(C)
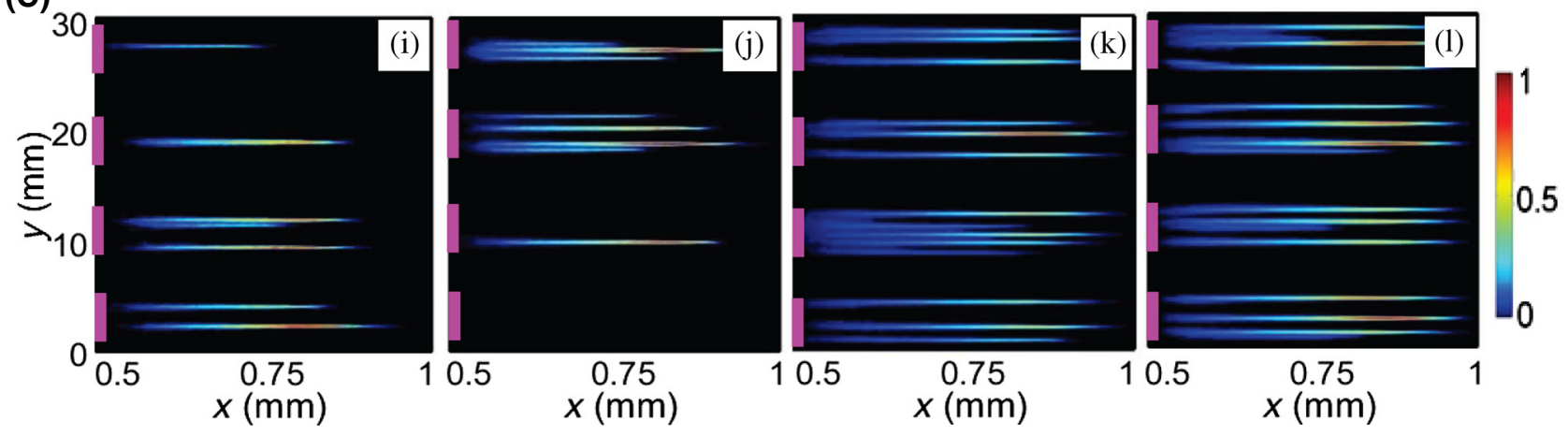

FIG. 2. Filamentary structures observed in experiment (row A), the corresponding pair correlation functions (row B), and PIC simulation results (row C) with increasing voltage. In the experiment, (a) $U=1.03 \mathrm{kV}$, (b) $U=1.13 \mathrm{kV}$, (c) $U=1.15 \mathrm{kV}$, and (d) $U=1.21 \mathrm{kV}$. The frequency of the applied voltage is $f=53 \mathrm{kHz}$. The working gas is $85 \%$ He and $15 \%$ air. The gas pressure is $p=350$ Torr, the gas gap is $d=1.4 \mathrm{~mm}$. The thin white circles indicate the cells of the lattice template. In each bar chart on the top, the numbers 2 to 5 denote the number of the filaments in each cell, while the percentage gives their proportion over the total cells. The blue dashed lines in row (B) indicates the maximum peak of each curve. In simulation (C), the maximum electron density distributions of filamentary discharges are shown. The parameters are (i) $U=0.7 \mathrm{kV}, t=1.18 \mu \mathrm{s} ;$ (j) $U=0.9 \mathrm{kV}, t=1.07 \mu \mathrm{s} ;(\mathrm{k}) U=1.3 \mathrm{kV}, t=0.89 \mu \mathrm{s}$; (l) $U=1.5 \mathrm{kV}, t=0.81 \mu \mathrm{s}$. A normalized color bar is used here, where the maximum electron densities are $3 \times 10^{18} \mathrm{~m}^{-3}, 6 \times 10^{18} \mathrm{~m}^{-3}$, $1.8 \times 10^{19} \mathrm{~m}^{-3}$, and $2 \times 10^{19} \mathrm{~m}^{-3}$ for the four cases, respectively. The purple bars on the left side of each figure denote the positions of the electrode segments.

(A)] reveals that the number of filaments increases as the voltage is raised. Only one or a pair of filaments are generated in each cell when the voltage is low [Fig. 2(a)], while localized triplets [Fig. 2(b)] and squares or pentagons [Figs. 2(c) and 2(d)] in each cell are produced when the voltage is sufficiently high. In addition, the filaments are prone to form near the border of the electrode cell when the number of filaments in each cell is greater than one. However, if there is only a single filament generated in the cell, its position could be either in the middle or near the border of the cells. Further, to analyze the spatial order of these filamentary structures, we calculate the pair correlation functions from the discharge density distributions. The pair correlation functions have been used to characterize complex plasma crystals [33,34]. The results obtained in the present case are shown in row (B) in Fig. 2. Here, $\rho_{r}$ is the probability density to find a filament at distance $r$ from a given filament. It is shown that $\rho_{r}$ has maximum peaks at $r_{m}=2.70 \mathrm{~mm}, 2.53 \mathrm{~mm}, 2.51 \mathrm{~mm}$, and $2.01 \mathrm{~mm}$ in (e)-(h), respectively, which gives the distances with the largest probability for formation of pairs of filaments. 
For the simulation results shown in row (C) of Fig. 2, one can see that some individual filaments are formed in each segment of the electrode. Here, the electron density distributions can be considered as the luminous filaments that are usually observed in experiments. The development of the filaments can be described as follows. First, the initial charged particles in the gas gap move quickly toward the electrodes under the influence of the electric field. The energetic ions, which strike on the dielectric layer, will induce secondary electrons that are fed into the discharge channel as the seed charges and impactionization avalanches occur, leading to the formation of discharge filaments. Typically, the discharge undergoes three different phases: the Townsend phase, the spacecharge-dominated avalanche phase, and the decay phase [32]. Here, it is evident that the number of filaments generated in each segment increases with the applied voltage from $U=0.7 \mathrm{kV}$ to $1.5 \mathrm{kV}$ as shown in Figs. 2(i)-2(1). Multiple triplets that distribute periodically emerge at the high voltage [Fig. 2(1)]. The filaments are easier to form close to the boundaries of the electrode segment when more than one filament is ignited due to the remarkable electric field distortion at these positions. However, when there is only one filament initiated on the segment, it can be located either in the middle or near the boundaries of the segment. The simulation results are consistent with the experimental observations. Moreover, the electron density of filaments can be obtained here, which is of the order of $10^{18}-10^{19} \mathrm{~m}^{-3}$. This can be used to estimate the plasma frequency as well as the plasma dielectric constant, which are essential to examine the photonic band gaps. Note that a higher voltage frequency and a shorter gas-gap distance are adopted in simulation as compared to the experiment in order to save computational resources. Owing to the fact that the collision frequencies between the charged particles and neutrals (approximately $10^{1}-10^{2} \mathrm{GHz}$ ) are much larger than the frequency of the applied voltage (approximately $10^{1}-10^{2} \mathrm{kHz}$ ), it is expected that the fundamental discharging process and trends that we discuss here are similar in both the simulation and experiment.

A rich variety of plasma lattice structures including complex superlattices are obtained in our experiment when changing the discharge parameters (Fig. 3). In the following, we show some examples of different plasma lattice structures' formation. First, the spatial symmetries of plasma lattices can be completely changed with an increase of the applied voltage for a fixed gas pressure and gas composition. As shown in Fig. 3(a), a triangular lattice is formed at the voltage $U=2.24 \mathrm{kV}$. The corresponding pair correlation function $\rho_{r}$ has a maximum peak at $r_{m}=3.37 \mathrm{~mm}$. When the voltage is increased to $U=2.32 \mathrm{kV}$, a triangular superlattice is formed, which is composed of the large and small spots. At $U=2.88 \mathrm{kV}$, a hexagonal lattice is formed with $r_{m}=2.78 \mathrm{~mm}$, where the corresponding correlation function is shown in
Fig. 3( $\left.a_{4}\right)$. Correspondingly, their photonic band diagrams will change with the symmetry variation. It has been shown that the formation of superlattices can give rise to large band gaps $[3,4]$. Second, under a different gas pressure and different composites, the lattice constant of plasma lattice structures can be modified. As presented in Fig. 3(b), a hexagon lattice is formed at a relatively low voltage of $U=2.34 \mathrm{kV}$, whose lattice constant is $a \approx$ $r_{m}=3.79 \mathrm{~mm}$. For $U=2.65 \mathrm{kV}$, the hexagonal lattice becomes more regular and the lattice constant is decreased to $a \approx r_{m}=3.51 \mathrm{~mm}$. For $U=2.84 \mathrm{kV}$, more filaments are produced, and therefore, a well-defined hexagonal lattice with a small lattice constant is formed in which $a \approx$ $r_{m}=2.57 \mathrm{~mm}$, where the corresponding correlation function is shown in Fig. 3(b $\left.b_{4}\right)$. Finally, Fig. 3(c) shows a case when the lattice structure remains relatively stable while the plasma densities are changed under different voltages. For a given voltage, the bright filaments (with plasma density $n_{1}$ ) form near the border, weak discharge regions (with plasma density $n_{2}$ ) occur in the middle of the electrode cells, and uniform plasma (with plasma density $n_{3}$ ) is generated in the space between cells as the background. In this case, the band diagrams of plasma lattice structures can be modulated by changing these three plasma densities (corresponding to three different permittivities), which are controlled by the applied voltages. This may provide additional flexibility for band diagram control of plasma lattice structures. One notes that both PCs or PPCs are, so far, generally fabricated with an alternation of two different permittivities, which is restricted by the current manufacturing techniques since it is much more complicated to introduce different permittivities in structures.

Interestingly, the plasma lattice structures shown above not only allow for active control in their spatial structures, but also can be modulated in time. As presented in Fig. 4, for simple structures, only a single current peak emerges in each half cycle of the applied voltage. This indicates that all of the filaments are ignited simultaneously and they repeat in the same manner in the following half cycles. The plasma lattice structures in this case exist in a time window of about $0.15 \mu \mathrm{s}$ in full width at half maximum (FWHM). The jitter of the current spike appearance at each half period of applied voltage is generally less than $15 \mathrm{~ns}$. Therefore, by changing the frequency of the applied voltage, the formation of plasma lattice structures can be temporally controlled. The lifetime of these plasma lattice structures is long enough to be applied to control short-pulsed microwaves, which can be as short as sub-nanoseconds. Such pulsed microwaves show a variety of applications, including precision radar range finding, precision radiolocation, wideband communications, time-resolved holographic imaging, signal processing, pulsed microwave generation and detection, and so on. [35-41]. 
(a) $2.24 \mathrm{kV}$

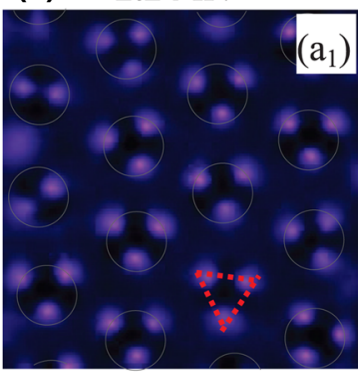

(b) $2.34 \mathrm{kV}$

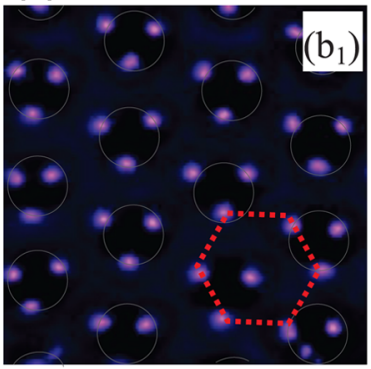

(c) $1.94 \mathrm{kV}$

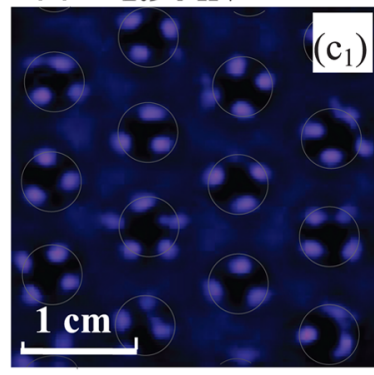

$2.32 \mathrm{kV}$

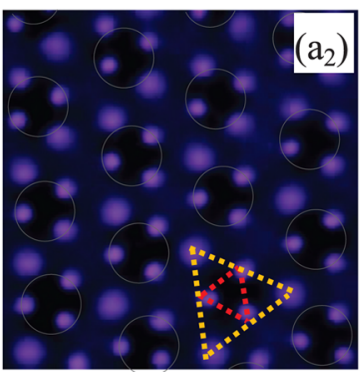

$2.65 \mathrm{kV}$

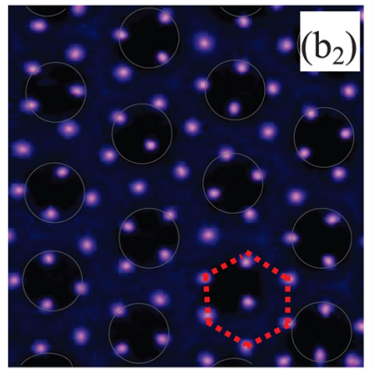

$2.04 \mathrm{kV}$

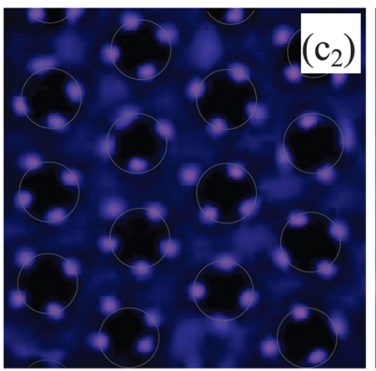

\section{$2.88 \mathrm{kV}$}

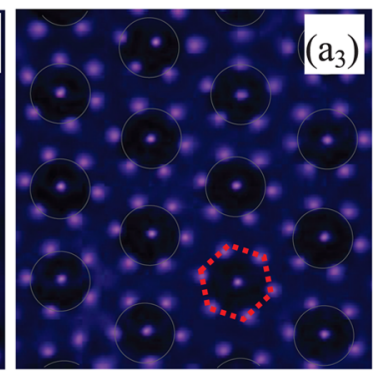

$2.84 \mathrm{kV}$

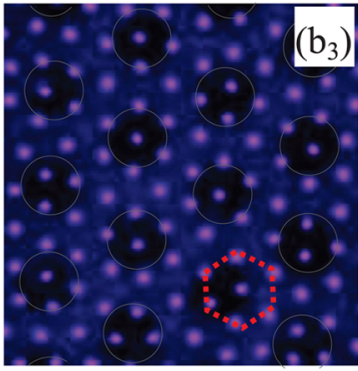

$2.22 \mathrm{kV}$

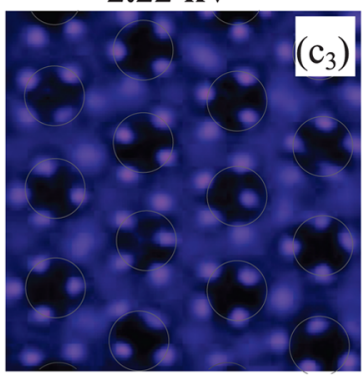

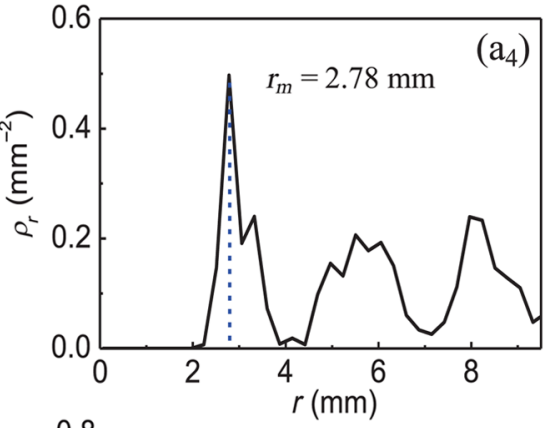
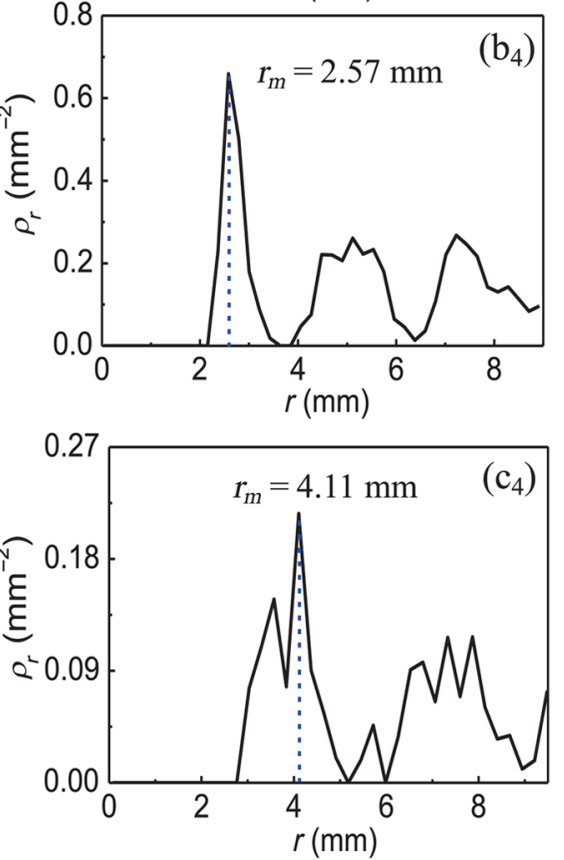

FIG. 3. Experimental observations of plasma lattice structures obtained with increasing voltage and different gas compositions. (a) $70 \%$ argon and $30 \%$ air, $p=200$ Torr, $d=1.4 \mathrm{~mm}$; (b) $10 \% \mathrm{He}$ and $90 \%$ air, $p=150$ Torr, $d=1.4 \mathrm{~mm}$; (c) $50 \% \mathrm{He}$ and $50 \%$ air, $p=150$ Torr, $d=1.4 \mathrm{~mm}$. The frequency of the applied voltage is $f=53 \mathrm{kHz}$. The basic symmetries of the structures are denoted by dashed lines and the discharge cells are marked with thin gray circles. Plots $\left(\mathrm{a}_{4}\right),\left(\mathrm{b}_{4}\right)$, and $\left(\mathrm{c}_{4}\right)$ show the corresponding pair correlation functions for the patterns given in $\left(a_{3}\right),\left(b_{3}\right)$, and $\left(c_{3}\right)$, respectively. The blue dashed lines indicate the maximum peak of each curve. In $\left(c_{3}\right)$, only the bright discrete spots are identified and used to calculate the pair correlation functions.

For complex plasma lattice structures, they are generally time averaged and composed of multiple dynamic substructures. This results in the time-modulated photonic band diagrams, since each substructure corresponds to a unique dispersion relation. Figure 5 demonstrates the spatial-temporal-resolved measurements of the hexagonal lattice by using fast camera diagnostics. The two current pulses marked by the red and green areas suggest that the discharge ignition takes place twice in each half cycle of the applied voltage. During the first pulse, the hexagon sublattice $\left(H_{1}\right)$ emerges in a time window of about $0.17 \mu \mathrm{s}$ in FWHM with a lattice constant of $a \approx 3.70 \mathrm{~mm}$. During the second pulse, the honeycomb substructure $\left(\mathrm{H}_{2}\right)$ is produced in a time window of about $0.18 \mu$ s in FWHM. Thus, the hexagonal crystal is actually a transient structure composed of the hexagonal sublattice and the honeycomb sublattice, which emerge with the sequence of $H_{1}-H_{2}-H_{1}-H_{2}$ in each cycle of the applied voltage. This is a result of the nonlinear effect, since some of the eigenmodes of the system are spontaneously selected, giving rise to spatiotemporally stable plasma structures $[42,43]$. The good periodic repeatability facilitates the synchronization between the plasma lattice structures and the input microwave sources in practical applications, even if the microwave sources have durations as short as a few nanoseconds [35,41]. The hexagon and honeycomb are two important topology structures in photonic crystals for producing large band gaps. Since these two substructures have very different dispersion relations, a time-modulated photonic band diagram can be realized 

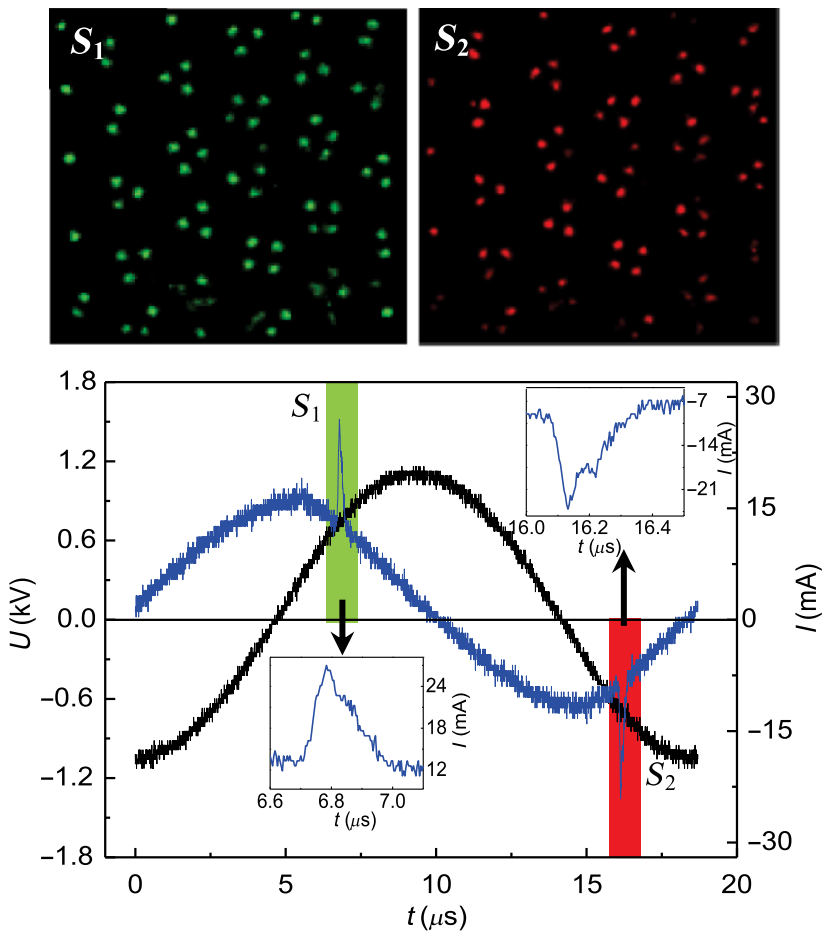

FIG. 4. Spatiotemporally resolved filamentary structures corresponding to two discharge current peaks $\left(S_{1}, S_{2}\right)$ are shown, obtained using fast camera diagnostics, together with the oscillogram of the discharge current $I$ (blue line) and the supply voltage $U$ (black line), where $U=1.15 \mathrm{kV}, f=53 \mathrm{kHz}, p=350$ Torr, $d=1.4 \mathrm{~mm}$, and the working gas is $85 \% \mathrm{He}$ and $15 \%$ air. These correspond to the parameters in Fig. 2(c). The insets are enlargements of the discharge current spikes.

in the way proposed here. Similarly, by changing the frequency of the applied voltage, the time period of plasma lattice structures can be dynamically controlled.

We emphasize that the plasma lattice structures suggested here can be tuned flexibly over a wide range by changing different discharge parameters, including the structure of the template, ac voltage, gas pressure, and compositions, and so on. The fundamental symmetry of plasma lattice structures is determined by the mesh template. The plasma filaments, which are selforganized into different fine structures in each discharge unit, modify the fundamental plasma lattice structures, changing their lattice constants or forming diverse superlattices. This provides a direct way of giving preference to certain symmetries of the plasma lattice structures as compared with the ordinary planar DBD systems. Moreover, the lattice constants can be adjusted from millimeters to centimeters, and the electron density can be adjusted between $10^{18}-10^{21} \mathrm{~m}^{-3}$, which corresponds to the electron plasma frequency of $\omega_{\text {pe }} / 2 \pi=9-283 \mathrm{GHz}$. With these plasma lattice structures, photonic band gaps can be formed when the structural parameters are appropriately selected. For example, for the hexagon sublattice
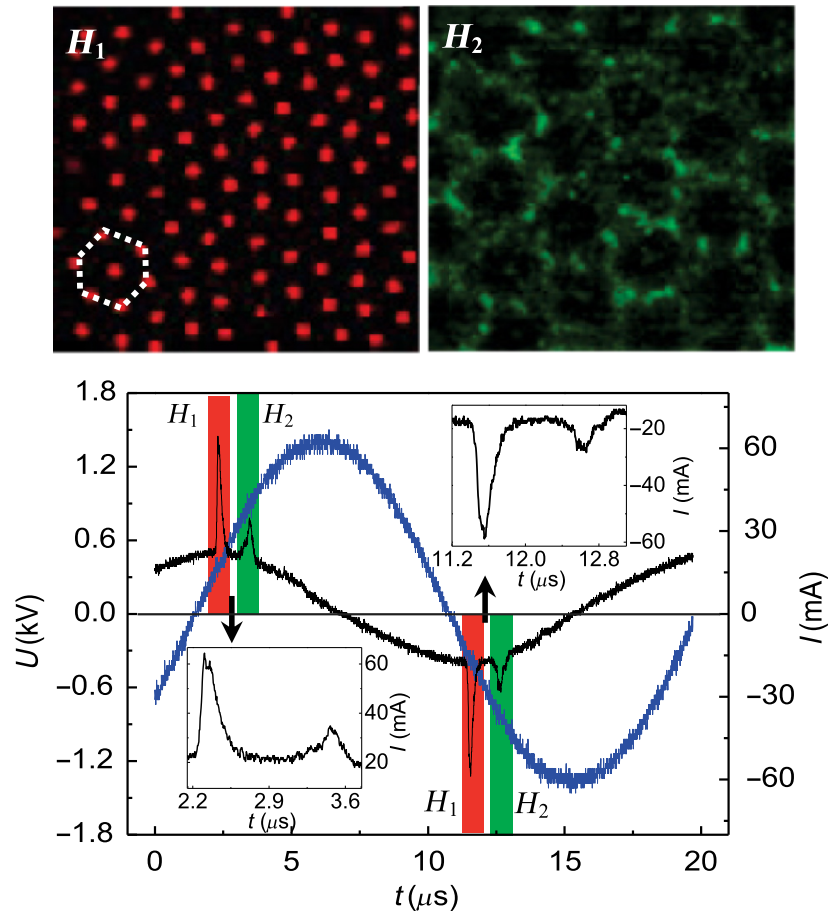

FIG. 5. Spatiotemporally resolved measurements of the hexagon lattice by using fast camera diagnostics together with the waveforms of the voltage and current. The hexagon sublattice corresponding to the first current pulse is indicated with $H_{1}$, while the honeycomb sublattice corresponding to the second current pulse is $H_{2}$. The adopted parameters are $U=1.45 \mathrm{kV}$, $f=53 \mathrm{kHz}, 10 \%$ air and $90 \% \mathrm{He}, p=380$ Torr, $d=1.4 \mathrm{~mm}$. The insets are enlargements of the discharge current spikes.

shown in Fig. $5\left(H_{1}\right)$, the real part of the plasma dielectric constant $\operatorname{Re}\left(\varepsilon_{p}\right) \sim 0.83$, when the frequency of probing electromagnetic waves $\omega / 2 \pi=40 \mathrm{GHz}$ with $\lambda / a \approx 2$, the electron density $n_{e}=10^{19} \mathrm{~m}^{-3}$, and the elastic collision frequency between electrons and neutral atoms $v_{m} /$ $2 \pi=57 \mathrm{GHz}$. To some extent, this is similar to the plasma photonic crystals studied by Sakai et al. by utilizing an array of facing capillary electrode pairs [11,12], where clear band gaps between $60-63 \mathrm{GHz}$ were detected in their experiment for plasma structures with $n_{e}=10^{19} \mathrm{~m}^{-3}$, $a=2.5 \mathrm{~mm}$, and $p=200$ Torr. Moreover, photonic band gaps were observed for a square plasma structure when $\operatorname{Re}\left(\varepsilon_{p}\right) \sim 0.8-0.9$ with the plane-wave expansion method [11]. Consequently, the plasma lattice structures presented here are potential plasma photonic crystals for microwaves at wavelengths from $\mathrm{mm}$ to $\mathrm{cm}$.

\section{CONCLUSION}

In summary, we experimentally demonstrate the generation of spatiotemporally controllable plasma lattice structures in DBD. By introducing a latticed water electrode into the planar system, a variety of plasma lattice structures including complex superlattices are obtained. 
By readily changing the symmetry of the electrode lattice, the filamentary microstructure in each electrode unit, and the plasma density, various plasma lattices with different symmetries can be dynamically constructed. Twodimensional PIC MCC simulations are carried out to study the formation of plasma structures, which show the controllability of plasma filament formation in DBD with the latticed electrode, in good agreement with our experimental observation. In addition, we show that the plasma lattices have a typical lifetime of about $\mu$ s under our discharge conditions, which is long enough to manipulate short microwave pulses. Our work suggests an effective way to realize spatiotemporally controllable plasma lattice structures, which may find broad applications in the manipulation of microwaves.

\section{ACKNOWLEDGMENTS}

This work was supported by the National Natural Science Foundation of China (Grants No. 11875014, No. 11721091, No. 11505044, and No. 11655002), the Natural Science Foundation of Hebei Province (Grants No. A2016201066 and No. A2017201099), and the Research Foundation of Education Bureau of Hebei Province (Grants No. BJ2016006 and No. ZD2015025). W. L. Fan wishes to thank Dr. Fucheng Liu and Dr. Yafeng He for helpful discussions.

[1] E. Yablonovitch, Inhibited Spontaneous Emission in SolidState Physics and Electronics, Phys. Rev. Lett. 58, 2059 (1987).

[2] S. John, Strong Localization of Photons in Certain Disordered Dielectric Superlattices, Phys. Rev. Lett. 58, 2486 (1987).

[3] C. M. Anderson and K. P. Giapis, Larger Two-Dimensional Photonic Band Gaps, Phys. Rev. Lett. 77, 2949 (1996).

[4] H. F. Zhang and Y. Q. Chen, The properties of twodimensional fractal plasma photonic crystals with ThueMorse sequence, Phys. Plasmas 24, 042116 (2017).

[5] J. Kitagawa, M. Kodama, S. Koya, Y. Nishifuji, D. Armand, and Y. Kadoya, $\mathrm{THz}$ wave propagation in two-dimensional metallic photonic crystal with mechanically tunable photonic-bands, Opt. Express 20, 17271 (2012).

[6] H. M. H. Chong and R. M. De La Rue, Tuning of photonic crystal waveguide microcavity by thermooptic effect, IEEE Photonics Technol. Lett. 16, 1528 (2004).

[7] D. Yang, H. Tian, and Y. Ji, Nanoscale photonic crystal sensor arrays on monolithic substrates using side-coupled resonant cavity arrays, Opt. Express 19, 20023 (2011).

[8] W. Park and J. B. Lee, Mechanically tunable photonic crystal structure, Appl. Phys. Lett. 85, 4845 (2004).

[9] B. Guo, M. Xie, and L. Peng, Photonic band structures of one-dimensional photonic crystals doped with plasma, Phys. Plasmas 19, 072111 (2012).

[10] O. Sakai, T. Sakaguchi, and K. Tachibana, Verification of a plasma photonic crystal for microwaves of millimeter wavelength range using two-dimensional array of columnar microplasmas, Appl. Phys. Lett. 87, 241505 (2005).

[11] O. Sakai, T. Sakaguchi, and K. Tachibana, Photonic bands in two-dimensional microplasma arrays. I. Theoretical derivation of band structures of electromagnetic waves, J. Appl. Phys. 101, 073304 (2007).

[12] T. Sakaguchi, O. Sakai, and K. Tachibana, Photonic bands in two-dimensional microplasma arrays. II. Band gaps observed in millimeter and subterahertz ranges, J. Appl. Phys. 101, 073305 (2007).

[13] O. Sakai, S. Yamaguchi, A. Bambina, A. Iwai, Y. Nakamura, Y. Tamayama, and S. Miyagi, Plasma metamaterials as cloaking and nonlinear media, Plasma Phys. Control. Fusion 59, 014042 (2017).

[14] B. Wang and M. A. Cappelli, A plasma photonic crystal bandgap device, Appl. Phys. Lett. 108, 161101 (2016).

[15] L. Zhang and J. T. Ouyang, Experiment and simulation on one-dimensional plasma photonic crystals, Phys. Plasmas 21, 103514 (2014).

[16] E. H. Matlis, T. C. Corke, B. Neiswander, and A. J. Hoffman, Electromagnetic wave transmittance control using self-organized plasma lattice metamaterial, J. Appl. Phys. 124, 093104 (2018).

[17] M. K. Chaudhari and S. Chaudhari, Tuning photonic bands in plasma metallic photonic crystals, Phys. Plasmas 23, 112118 (2016).

[18] T. F. Khalkhali and A. Bananej, Effect of shape of scatterers and plasma frequency on the complete photonic band gap properties of two-dimensional dielectric-plasma photonic crystals, Phys. Lett. A 380, 4092 (2016).

[19] B. Wang and M. A. Cappelli, A tunable microwave plasma photonic crystal filter, Appl. Phys. Lett. 107, 171107 (2015).

[20] S. Parsons, J. Gregório, and J. Hopwood, Microwave plasma formation within a 2D photonic crystal, Plasma Sources Sci. Technol. 26, 055002 (2017).

[21] J. Gregório, S. Parsons, and J. Hopwood, Reconfigurable photonic crystal using self-initiated gas breakdown, Plasma Sources Sci. Technol. 26, 02LT03 (2017).

[22] Z. M. Sheng, J. Zhang, and D. Umstadter, Plasma density gratings induced by intersecting laser pulses in underdense plasmas, Appl. Phys. B 77, 673 (2003).

[23] L. L. Yu, Z. M. Sheng, and J. Zhang, Plasma Bragg density gratings produced by optical-field ionization, J. Opt. Soc. Am. B 26, 2095 (2009).

[24] G. Lehmann and K. H. Spatschek, Transient Plasma Photonic Crystals for High-Power Lasers, Phys. Rev. Lett. 116, 225002 (2016).

[25] Y. P. Bliokh, J. Felsteiner, and Y. Z. Slutsker, Total Absorption of an Electromagnetic Wave by an Overdense Plasma, Phys. Rev. Lett. 95, 165003 (2005).

[26] W. L. Fan, X. C. Zhang, and L. F. Dong, Two-dimensional plasma photonic crystals in dielectric barrier discharge, Phys. Plasmas 17, 113501 (2010).

[27] L. F. Dong, Y. F. He, W. L. Liu, R. L. Gao, H. F. Wang, and H. T. Zhao, Hexagon and square patterned air discharges, Appl. Phys. Lett. 90, 031504 (2007).

[28] J. P. Verboncoeur, A. B. Langdon, and N. T. Gladd, An object-oriented electromagnetic PIC code, Comput. Phys. Commun. 87, 199 (1995). 
[29] V. Vahedi and M. Surendra, A Monte Carlo collision model for the particle-in-cell method: applications to argon and oxygen discharges, Comp. Phys. Comm. 87, 179 (1995).

[30] C. K. Birdsall, Particle-in-Cell Charged-Particle Simulations, plus Monte Carlo Collisions with neutral Atoms, PIC-MCC, IEEE Trans Plasma Sci. 19, 65 (1991).

[31] J. P. Verboncoeur, Particle simulation of plasmas: review and advances, Plasma Phys. Control Fusion 47, A231 (2005).

[32] W. L. Fan, Z. M. Sheng, X. X. Zhong, W. M. Wang, Y. T. Li, and J. Zhang, Particle simulation of filamentary structure formation in dielectric barrier discharge, Appl. Phys. Lett. 102, 094103 (2013).

[33] C. Strümpel and H.-G. Purwins, Spatiotemporal filamentary patterns in a dc-driven planar gas discharge system, Phys. Rev. E 63, 026409 (2001).

[34] A. Melzer, A. Homann, and A. Piel, Experimental investigation of the melting transition of the plasma crystal, Phys. Rev. E 53, 2757 (1996).

[35] J. Shiloh, B. Mandelbaum, and E. Heyman (Eds.), UltraWideband Short-Pulse Electromagnetics 4 (Springer, New York, 2002).

[36] C. A. Sullivan, W. W. Destler, J. Rodgers, and Z. Segalov, Short-pulse high-power microwave propagation in the atmosphere, J. Appl. Phys. 63, 5228 (1988).
[37] M. Gatzke, B. Broers, L. D. Noordam, R. B. Watkins, and T. F. Gallagher, Short-pulse microwave ionization of $\mathrm{Na}$ Rydberg atoms, Phys. Rev. A 50, 2502 (1994).

[38] D. W. Scholfield, J. M. Gahl, and B. W. Mullins, Investigation of the Paschen curve of nitrogen via the application of nanosecond pulsed electromagnetic radiation, J. Appl. Phys. 76, 1469 (1998).

[39] J. Lester, J. M. Gahl, D. W. Scholfield, and N. Shimomura, Investigation of intense electromagnetic transient phenomenon and Paschen curves for hydrogen and helium in subnanosecond regime, IEEE Trans. Plasma Sci. 28, 496 (2000).

[40] T. E. McEwan, Short pulse microwave transceiver, United States Patent No.: US 6191724B1 (2001).

[41] C. Chang, C. Wu, Y. K. Pu, M. Zhu, X. Zhang, and J. Verboncoeur, Diagnostic of ultrafast temporal plasma evolution in high-power microwave discharge, J. Appl. Phys. 121, 213301 (2017).

[42] H. G. Purwins, H. U. Bodecker, and S. Aminarashvili, Dissipative solitons, Adv. Phys.. 59, 485 (2010).

[43] H. G. Purwins and L. Stollenwerk, Synergetic aspects of gas-discharge: lateral patterns in dc systems with a high ohmic barrier, Plasma Phys. Control. Fusion 56, 123001 (2014). 